\title{
Pricing Strategy of Dual Channel Supply Chain under Asymmetric Information
}

\author{
Zhou Yongwei ${ }^{1,2, *}$ and Fan Hehua ${ }^{1}$ \\ ${ }^{1}$ College of Science, Zhengzhou University of Aeronautics Zhengzhou, China, \\ 450015; \\ ${ }^{2}$ Collaborative innovation center for aviation economic development of Henan \\ province, Zhengzhou, China, 450015 \\ Corresponding author: ZHOU Yong-wei, zwwfhh@126.com
}

\begin{abstract}
Aiming at the dual channel supply chain which sells a single product, and composed of the retailer as the leader and the manufacturer as the follower, firstly, by establishing the Stackelberg game model, we study the manufacturer and the retailer's optimal pricing strategy under the condition of retailer's innovation cost coefficient is common knowledge, then further analyze the manufacturer and the retailer's optimal pricing strategy under the retailer's innovation cost coefficient is his own private information, and discuss the existence condition of perfect Bayesian Nash equilibrium pricing. The research result showed that under the condition of asymmetric information, even the manufacturer has belief revision, his optimal pricing is always consistent with the type of retailer's optimal pricing, but there are several pricing strategy choices to retailer. Under different parameters' values, the dynamic game model with asymmetric information possibly exist separating equilibrium, pooling equilibrium, and semi-separating equilibrium.
\end{abstract}

Keywords: Asymmetric information; dual channel; innovation cost; pricing strategy

\section{Introduction}

With the rapid development of internet technology and the third-part logistics, more and more brand manufacturers have begun to set up direct sales by introducing internet channel, such as IBM, HP and so on [1], they all obtained a great success by the dual channel strategy. But the introduce of direct channel also give rise to the channel conflict and the benefit game between the manufacturer and the retailer, thus it lowers the efficiency of supply chain, Webb [2] pointed out that product pricing is the primary factor of channel conflict, so the pricing strategy is an important issue of dual channel supply chain.

There have many scholars studied the pricing problem of dual channel supply chain, and obtained abundant research achievements, the earlier scholar is Chiang W K [3], he discussed the pricing of dual channel supply chain and the influence of direct channel on retail channel, the research result showed that the internet channel may affect the pricing of retailer, thereby it lowered the double marginalization of dual channel supply chain, and improved the total profit of supply chain. Cattani et al [4] studied the manufacturer's pricing problem of dual channel supply chain by use of consumer utility theory. Aray's[5] researches indicated that when the manufacturer has production cost advantage and the retailer has distribution cost advantage, the added direct channel can realize the Pareto improvement of manufacturer and retailer in supply chain. Cai [6] et al discussed the dual channel supply chain's equilibrium pricing under the condition of consistent and inconsistent price, and pointed out that price discount contract can improve the 
performance of supply chain. Zhang Xiaojuan, Wang Yong [7] studied the dual channel supply chain's pricing strategy with capital constraint of retailer, it showed that the deferred payment model not only increase the retailer's profit, but also raise the competitiveness in market. Shen Chengran et al [8] discussed the dual channel supply chain's pricing strategy under price comparison behavior of consumers, the result showed that under the price comparison behavior, the retailer's benefit lost, and intensified the channel conflict. Zeng Mingang [9] studied the dual channel supply chain's pricing strategy under the condition of random demand, it showed that the consumer's channel preference coefficient, demand uncertainty, marginal cost of production and so on, are all have certain influence relationship on the supply chain's pricing. Fan Hehua et al [10] studied the dual channel supply chain's optimal pricing strategy under the uncertainty demand and the capital constraint of retailer. Xu Minli et al [11] studied the pricing decision of dual channel supply chain with different risk preference based on the CVaR criterion, it is found that the manufacture and retailer will adopt different pricing strategy according to their different risk preference. Zhou Y W et al [12] discussed the dual channel supply chain's pricing problem under the condition of consumers' channel preference, and realized the Pareto improvement of manufacturer and retailer by a price mark-up contract. But the above mentioned references are discussed under the condition of perfect information.

Concrete to asymmetric information, Chen Jun et al [13] studied the incentive mechanism for different service levels provided by retailer on the influence of supply chain's performance, the conclusions implied that the manufacturer's profit decreases under asymmetric information, and it also lowered the whole supply chain system's performance. Aiming at the dual channel supply chain system with the manufacturer as the follower, Tian Wei, Jiang Kan [14] studied the influence of retailer's innovation ability asymmetric information on the supply chain's profit. Wang Hong et al $[15,16]$ further studied the dual channel supply chain system composed of one risk aversion manufacturer and one risk aversion retailer, and analyzed the supply chain's optimal pricing strategy under the condition of the manufacturer's risk averse value is his own private information.

In fact, in the dual channel supply chain, as the introducing of direct channel, it will inevitably bring out the competition to the traditional retail channel, in addition, as the manufacturer has its cost advantage, as for the retailer, how to lower its distribution cost and improve its competitiveness by constantly innovation is the key of obtaining survival and development. Different to above mentioned document researches, the article studied the dual channel supply chain which sells a single product, and composed of the retailer as the leader and the manufacturer as the follower, and in the premise that the retailer's innovation input cost coefficient is his own private information, it mainly focused on the manufacturer and the retailer's optimal pricing strategy of the dual channel supply chain under the asymmetric information circumstance.

\section{Model Description}

The article considered the dual channel supply chain which sells a single product, and composed of one retailer and one manufacturer, it assumed that the manufacturer's marginal cost of producing the unit product is 0 , in addition, for the sake of discussion, it also assumed that manufacturer's distribution cost in two channels is $0[17,18]$. In the traditional retail channel, the manufacturer's unit wholesale price to retailer is $w$, then the retailer sell the products to customers with a certain marginal distribution cost. The demand functions of two channels are denoted by [19]:

$$
\begin{gathered}
Q_{d}=(\gamma+\rho) A-\alpha_{d} p_{d}+\beta p_{r}, \\
Q_{r}=(1-\gamma) A-\alpha_{r} p_{r}+\beta p_{d},
\end{gathered}
$$


respectively.

Where $Q_{r}$ and $Q_{d}$ represent the market demand of retail channel and direct channel, $A$ is the total market demand, $\gamma$ is the proportion of market demand in direct channel, $\rho$ is the proportion of potential demand attracted by the dual channel sales mode, $p_{r}, p_{d}$ are the sale price in traditional retail channel and direct channel, respectively, $\alpha_{r}, \alpha_{d}$ are the price elasticity of retail channel and direct channel, respectively, $\beta$ is the two channels' cross price elasticity, and satisfies $\alpha_{r}>\alpha_{d}>\beta$.

It is assumed that the manufacturer's unit wholesale price $w$ to retailer has determined by contract, and the retailer is the leader, the manufacturer is the follower, all the parameters are common knowledge, the article mainly discuss the dual channel supply chain's pricing strategy under asymmetric information, the inventory problem are not concerned, similar to [15], suppose that the retailer place order after he observed the market demand, the manufacturer adopts the way of Make-to-Order production, and the retailer cannot buy products from direct channel.

To improve the competitiveness in markets, in dual channel supply chain circumstance, the retailer innovate in the cost of logistics, inventory and so on, and lowers its marginal distribution cost by innovation input, similar to $[14,20]$, it assumed that the retailer's input $c(\theta)$ can bring down $\theta r$ in marginal distribution cost $c_{r}$, where $0 \leq \theta \leq 1, r$ is the maximum decrement of marginal distribution cost after the retailer's innovation input, it represents the retailer's innovation ability, $r<c_{r}$ shows that one part of the retailer's cost cannot be reduced at all. The retailer's innovation $\operatorname{cost}$ function $c(\theta)=\eta \theta^{2}$, the bigger $\theta$, the greater retailer's innovation input intensity, $\eta>0$ is the retailer's innovation cost coefficient, namely, the retailer can bring down $\theta r$ in marginal distribution cost by innovation input $\eta \theta^{2}$.

Then the manufacturer's profit is given by

The retailer's profit is given by

$$
\pi_{m}=p_{d} Q_{d}+w Q_{r},
$$

$$
\pi_{r}=\left(p_{r}-w-c_{r}+\theta r\right) Q_{r}-\eta \theta^{2},
$$

\section{The Pricing Model of Stackelberg Game under Perfect Information}

The article models the decision as a sequential, Stackelberg game, with the retailer as the leader and the manufacturer as the follower, the retailer's innovation cost coefficient $\eta$ is the common knowledge of two sides under the condition of perfect information, the retailer and manufacturer maximize their own profits respectively, analysis the manufacturer and retailer's profit function, we have the following theorem:

Theorem 1. If $8 \alpha_{d} \eta-\gamma^{2}\left(2 \alpha_{r} \alpha_{d}-\beta^{2}\right)>0$, the retailer's pricing decision exists the optimal solution.

Proof: The inverse order method is used to solve the Stackelberg equilibrium solution of supply chain system. Firstly, in order to maximize his profits, given the retailer's retail price $p_{r}$ and innovation input $\theta$, the manufacturer determines the direct channel price $p_{d}$.

Substituting (1), (2) into (3), and find the second-order partial derivative with respect to $p_{d}$, we obtained $\frac{\partial^{2} \pi_{m}}{\partial p_{d}{ }^{2}}=-2 \alpha_{d}<0$, so $\pi_{m}\left(p_{d}\right)$ is the second concave function of $p_{d}$, and by 


$$
\frac{\partial \pi_{m}}{\partial p_{d}}=\beta p_{r}-2 \alpha_{d} p_{d}+\beta w+A(\rho+\gamma)=0
$$

we obtained

$$
p_{d}\left(p_{r}, \theta\right)=\frac{\beta p_{r}+\beta w+A(\rho+\gamma)}{2 \alpha_{d}} .
$$

Substituting (2), (5) into (4), and find the second-order partial derivative with respect to $p_{r}$ and $\theta$, we obtained $\frac{\partial^{2} \pi_{r}}{\partial p_{r}{ }^{2}}=\frac{\beta^{2}}{\alpha_{d}}-2 \alpha_{r}<0, \frac{\partial^{2} \pi_{r}}{\partial \theta^{2}}=-2 \eta<0$. Therefore, there exists maximum on $\pi_{r}$, and by

$$
\left\{\begin{array}{l}
\frac{\partial \pi_{r}}{\partial p_{r}}=0 \\
\frac{\partial \pi_{r}}{\partial \theta}=0
\end{array}\right.
$$

we have

$$
\begin{gathered}
p_{r}^{*}=\frac{8 \alpha_{d}{ }^{2} \eta \xi_{1}-\gamma^{2}\left(2 \alpha_{r} \alpha_{d}-\beta^{2}\right) \xi_{2}}{\left(2 \alpha_{r} \alpha_{d}-\beta^{2}\right)\left[8 \alpha_{d} \eta-\gamma^{2}\left(2 \alpha_{r} \alpha_{d}-\beta^{2}\right)\right]}, \\
\theta^{*}=\frac{\xi_{3} \gamma}{8 \alpha_{d} \eta-\gamma^{2}\left(2 \alpha_{r} \alpha_{d}-\beta^{2}\right)},
\end{gathered}
$$

Where $\xi_{1}=A(1-\gamma)+\alpha_{r}\left(w+c_{r}\right)+4 \alpha_{d} \beta\left[A(\rho+\gamma)-c_{r} \beta\right]$,

$$
\begin{aligned}
& \xi_{2}=2 A \alpha_{d}(1-\gamma)+A \beta(\rho+\gamma)+\beta^{2} w \\
& \xi_{3}=2 A \alpha_{d}(1-\gamma)+A \beta(\rho+\gamma)+\beta^{2}\left(2 w+c_{r}\right)-2 \alpha_{r} \alpha_{d}\left(w+c_{r}\right) .
\end{aligned}
$$

Following we prove $\pi_{r}$ obtain the maximum on $\left(p_{r}^{*}, \theta^{*}\right)$, as $\frac{\partial^{2} \pi_{r}}{\partial p_{r}{ }^{2}}=\frac{\beta^{2}}{\alpha_{d}}-2 \alpha_{r}<0$, as its Hessian matrix [21]

$$
|H|=\left|\begin{array}{ll}
\left.\frac{\partial^{2} \pi_{r}}{\partial p_{r}{ }^{2}}\right|_{\left(p_{r}^{*}, \theta^{*}\right)} & \left.\frac{\partial^{2} \pi_{r}}{\partial p_{r} \partial \theta}\right|_{\left(p_{r}^{*}, \theta^{*}\right)} \\
\left.\frac{\partial^{2} \pi_{r}}{\partial \theta \partial p_{r}}\right|_{\left(p_{r}^{*}, \theta^{*}\right)} & \left.\frac{\partial^{2} \pi_{r}}{\partial \theta^{2}}\right|_{\left(p_{r}^{*}, \theta^{*}\right)}
\end{array}\right|=\frac{\left(2 \alpha_{r} \alpha_{d}-\beta^{2}\right)\left[8 \alpha_{d} \eta-\gamma^{2}\left(2 \alpha_{r} \alpha_{d}-\beta^{2}\right)\right]}{4 \alpha_{d}^{2}} .
$$

So, when $8 \alpha_{d} \eta-\gamma^{2}\left(2 \alpha_{r} \alpha_{d}-\beta^{2}\right)>0, \pi_{r}$ obtain the maximum on $\left(p_{r}{ }^{*}, \theta^{*}\right)$.

Substituting (7) into (5), we obtained

$$
p_{d}^{*}=\frac{\beta p_{r}^{*}+\beta w+A(\rho+\gamma)}{2 \alpha_{d}} .
$$

It is observed from (7), (8) and (9) that the retail price in retail channel increased with the increasing of innovation input cost coefficient, but the retailer's innovation input intensity decreased with the increasing of innovation input cost coefficient, the manufacturer's sale price in direct channel increased with the increasing of retail price in traditional retail channel, but it is not affected by the retailer's innovation input intensity. 


\section{Pricing Model of Stackelberg Game under Imperfect Information}

In the dual channel supply chain, as the retailer is the leader of Stackelberg game, the retailer can obtain all the information of supply chain, while the manufacturer is in weak position, he cannot fully aware of the retailer's private information, and it assumed that the retailer's innovation input cost coefficient is his private information, the manufacturer only know about the retailer's two types of high innovation cost $\eta^{H}$ and low innovation $\operatorname{cost} \eta^{L}$, and the retailer belongs to the two types' prior probability are $\mu\left(\eta^{H}\right)$ and $\mu\left(\eta^{L}\right)$, respectively, which is common knowledge. In order to make sense the problem, it assumed that neither the manufacturer can observe the retailer's innovation intensity $\theta$, otherwise the manufacturer's decision is the same to section 3 , and usually the innovation input need to proceed in advance, the retailer know his innovation cost information, the innovation intensity $\theta^{*}$ is constant for him.

Therefore, the manufacturer's pricing policy set is $\left\{p_{d}^{H}, p_{d}^{L}\right\}$, the retailer's pricing policy set is $\left\{p_{r}^{H}, p_{r}^{L}\right\}$, and $p_{d}^{H}<p_{d}^{L}, p_{r}^{H}<p_{r}^{L}$.

\subsection{The Manufacture's best Response Function}

As the manufacturer is the second mover, he can correct his prior probability after observed the retailer's action, it assumed that manufacturer's conditional probability about retailer are $\mu\left(p_{r}^{H} \mid \eta^{H}\right), \mu\left(p_{r}^{L} \mid \eta^{H}\right), \mu\left(p_{r}^{H} \mid \eta^{L}\right), \mu\left(p_{r}^{L} \mid \eta^{L}\right)$, respectively. The manufacturer can obtain the posterior probability by Bayes rule

$$
\begin{gathered}
\tilde{\mu}\left(\eta^{H} \mid p_{r}^{H}\right)=\frac{\mu\left(p_{r}^{H} \eta^{H}\right)}{\mu\left(p_{r}^{H}\right)}=\frac{\mu\left(\eta^{H}\right) \mu\left(p_{r}^{H} \mid \eta^{H}\right)}{\mu\left(\eta^{H}\right) \mu\left(p_{r}^{H} \mid \eta^{H}\right)+\mu\left(\eta^{L}\right) \mu\left(p_{r}^{H} \mid \eta^{L}\right)} \\
\tilde{\mu}\left(\eta^{L} \mid p_{r}^{H}\right)=1-\tilde{\mu}\left(\eta^{H} \mid p_{r}^{H}\right) \\
\tilde{\mu}\left(\eta^{H} \mid p_{r}^{L}\right)=\frac{\mu\left(p_{r}^{L} \eta^{H}\right)}{\mu\left(p_{r}^{L}\right)}=\frac{\mu\left(\eta^{H}\right) \mu\left(p_{r}^{L} \mid \eta^{H}\right)}{\mu\left(\eta^{H}\right) \mu\left(p_{r}^{L} \mid \eta^{H}\right)+\mu\left(\eta^{L}\right) \mu\left(p_{r}^{L} \mid \eta^{L}\right)} . \\
\tilde{\mu}\left(\eta^{L} \mid p_{r}^{L}\right)=1-\tilde{\mu}\left(\eta^{H} \mid p_{r}^{L}\right) .
\end{gathered}
$$

To the manufacturer's objective function:

When the manufacturer observed the retailer's retail price is $p_{r}^{H}$, which is corresponding to high innovation cost, the manufacturer's objective function is

$$
E\left(\pi_{m}\right)=\tilde{\mu}\left(\eta^{H} \mid p_{r}^{H}\right) \pi_{m}\left(\eta^{H}\right)+\left(1-\tilde{\mu}\left(\eta^{H} \mid p_{r}^{H}\right)\right) \pi_{m}\left(\eta^{L}\right) .
$$

When the manufacturer observed the retailer's retail price is $p_{r}^{L}$, which is corresponding to low innovation cost, the manufacturer's objective function is

$$
E\left(\pi_{m}\right)=\tilde{\mu}\left(\eta^{H} \mid p_{r}^{L}\right) \pi_{m}\left(\eta^{H}\right)+\left(1-\tilde{\mu}\left(\eta^{H} \mid p_{r}^{L}\right)\right) \pi_{m}\left(\eta^{L}\right) .
$$

While

$$
\pi_{m}\left(\eta^{H}\right)=\pi_{m}\left(\eta^{L}\right)=\pi_{m}=p_{d}\left[(\gamma+\rho) A-\alpha_{d} p_{d}+\beta p_{r}{ }^{H}\right]+w\left[(1-\gamma) A-\alpha_{r} p_{r}{ }^{H}+\beta p_{d}\right],
$$

so in (14) and (15)

$$
E\left(\pi_{m}\right)=\pi_{m}=p_{d}\left[(\gamma+\rho) A-\alpha_{d} p_{d}+\beta p_{r}{ }^{H}\right]+w\left[(1-\gamma) A-\alpha_{r} p_{r}{ }^{H}+\beta p_{d}\right] .
$$


It can be seen that the manufacturer's objective function is not directly related to the retailer's innovation cost coefficient; it is reflected by the retailer's decision variable, so when the manufacturer observed different direct sale prices, his response function form is similar to that of perfect information, whether the retailer's innovation cost coefficient is high or low, the manufacturer's action is consistent with that of retailer.

Therefore, the manufacturer's best response function to retailer is given by

$$
\begin{aligned}
p_{d}^{H}\left(p_{r}^{H}\right) & =\frac{\beta p_{r}^{H}+\beta w+A(\rho+\gamma)}{2 \alpha_{d}}, \\
p_{d}^{L}\left(p_{r}^{L}\right) & =\frac{\beta p_{r}^{L}+\beta w+A(\rho+\gamma)}{2 \alpha_{d}} .
\end{aligned}
$$

It is observed that no matter how the retailer's innovation cost coefficient, the manufacturer's optimal pricing is always keep consistent with the retailer, so it is unbelievable to the manufacturer's various kinds of threats.

\subsection{The Retailer's Pricing Decision}

The retailer can predict that his action will affect the manufacturer's action by posterior inference, so regardless of the type of retailer's innovation cost coefficient, as long as the high utility on $p_{r}^{H}$, he will choose $p_{r}^{H}$, or else he will choose $p_{r}^{L}$.

Form (7), we know that the retailer's polices $p_{r}^{H}$ and $p_{r}^{L}$ are

$$
\begin{aligned}
& p_{r}{ }^{H}=\frac{8 \alpha_{d}{ }^{2} \eta^{H} \xi_{1}-\gamma^{2}\left(2 \alpha_{r} \alpha_{d}-\beta^{2}\right) \xi_{2}}{\left(2 \alpha_{r} \alpha_{d}-\beta^{2}\right)\left[8 \alpha_{d} \eta^{H}-\gamma^{2}\left(2 \alpha_{r} \alpha_{d}-\beta^{2}\right)\right]}, \\
& p_{r}{ }^{L}=\frac{8 \alpha_{d}{ }^{2} \eta^{L} \xi_{1}-\gamma^{2}\left(2 \alpha_{r} \alpha_{d}-\beta^{2}\right) \xi_{2}}{\left(2 \alpha_{r} \alpha_{d}-\beta^{2}\right)\left[8 \alpha_{d} \eta^{L}-\gamma^{2}\left(2 \alpha_{r} \alpha_{d}-\beta^{2}\right)\right]},
\end{aligned}
$$

respectively.

And by $\eta^{H}>\eta^{L}$, we obtained $p_{r}^{H}>p_{r}^{L}$. That is to say, the retailer with high innovation cost will have a higher pricing.

(1) If the retailer belongs to the type of high innovation cost $\eta^{H}$, there are two

possible pricing policy of supply chain, they are $\left(p_{r}^{H}, \theta^{H}, p_{d}^{H}\left(p_{r}^{H}\right), \mid \eta^{H}\right)$ and $\left(p_{r}^{L}, \theta^{H}, p_{d}^{L}\left(p_{r}^{L}\right), \mid \eta^{H}\right)$, respectively, the retailer's profit are given by

$$
\begin{aligned}
& \pi_{r}\left(p_{r}^{H}, \theta^{H}, p_{d}^{H}\left(p_{r}^{H}\right) \mid \eta^{H}\right)=\left(p_{r}{ }^{H}-w-c_{r}+\theta^{H} r\right)\left[(1-\gamma) A-\alpha_{r} p_{r}{ }^{H}+\beta p_{d}{ }^{H}\right]-\eta^{H}\left(\theta^{H}\right)^{2} \\
& \pi_{r}\left(p_{r}^{L}, \theta^{H}, p_{d}^{L}\left(p_{r}^{L}\right), \mid \eta^{H}\right)=\left(p_{r}{ }^{L}-w-c_{r}+\theta^{H} r\right)\left[(1-\gamma) A-\alpha_{r} p_{r}{ }^{L}+\beta p_{d}{ }^{L}\right]-\eta^{H}\left(\theta^{H}\right)^{2}
\end{aligned}
$$

respectively.

Let

$$
\Delta \pi_{r}\left(\eta^{H}\right)=\pi_{r}\left(p_{r}^{H}, \theta^{H}, p_{d}^{H}\left(p_{r}^{H}\right) \mid \eta^{H}\right)-\pi_{r}\left(p_{r}^{L}, \theta^{H}, p_{d}^{L}\left(p_{r}^{L}\right), \mid \eta^{H}\right),
$$

and substituting (16)-(21) into $\Delta \pi_{r}\left(\eta^{H}\right)$, and obtained 


$$
\Delta \pi_{r}\left(\eta^{H}\right)=-\frac{r^{2}\left(\eta^{H}-\eta^{L}\right) \Sigma_{H}}{\left[8 \alpha_{d} \eta^{H}-\gamma^{2}\left(2 \alpha_{r} \alpha_{d}-\beta^{2}\right)\right]^{2}\left[8 \alpha_{d} \eta^{L}-\gamma^{2}\left(2 \alpha_{r} \alpha_{d}-\beta^{2}\right)\right]^{2}} .
$$

where $\Sigma_{H}=4 \alpha_{d}^{2} \alpha_{r}^{2} r^{4}-16 \alpha_{d}^{2} \alpha_{r} r^{2}\left(\eta^{H}+\eta^{L}\right)+64 \alpha_{d}^{2}\left(\eta^{L}\right)^{2}$

$$
-4 \alpha_{d} \alpha_{r} \beta^{2} r^{4}+8 \alpha_{d} \beta^{2} r^{2}\left(\eta^{H}+\eta^{L}\right)+\beta^{4} r^{4} \text {. }
$$

Therefore

If $\Sigma_{H}<0$, then $\Delta \pi_{r}\left(\eta^{H}\right)>0$, the retailer with high innovation cost will choose $p_{r}^{H}$;

If $\Sigma_{H}>0$, then $\Delta \pi_{r}\left(\eta^{H}\right)<0$, the retailer with high innovation cost will choose $p_{r}^{L}$;

If $\Sigma_{H}=0$, then $\Delta \pi_{r}\left(\eta^{H}\right)=0$, there is no difference in the two policies of the retailer with high innovation cost.

(2) If the retailer belongs to the type of low innovation cost $\eta^{L}$, there are two possible pricing policy of supply chain, they are $\left(p_{r}^{H}, \theta^{L}, p_{d}^{H}\left(p_{r}^{H}\right), \mid \eta^{L}\right)$ and $\left(p_{r}^{L}, \theta^{L}, p_{d}^{L}\left(p_{r}^{L}\right), \mid \eta^{L}\right)$, respectively, the retailer's profit are given by

$$
\begin{gathered}
\pi_{r}\left(p_{r}^{H}, \theta^{L}, p_{d}^{H}\left(p_{r}^{H}\right), \mid \eta^{L}\right)=\left(p_{r}{ }^{H}-w-c_{r}+\theta^{L} r\right)\left[(1-\gamma) A-\alpha_{r} p_{r}{ }^{H}+\beta p_{d}{ }^{H}\right]-\eta^{L}\left(\theta^{L}\right)^{2}, \\
\pi_{r}\left(p_{r}^{L}, \theta^{L}, p_{d}^{L}\left(p_{r}{ }^{L}\right), \mid \eta^{L}\right)=\left(p_{r}{ }^{L}-w-c_{r}+\theta^{L} r\right)\left[(1-\gamma) A-\alpha_{r} p_{r}{ }^{L}+\beta p_{d}{ }^{L}\right]-\eta^{L}\left(\theta^{L}\right)^{2}
\end{gathered}
$$

respectively.

Let

$$
\Delta \pi_{r}\left(\eta^{L}\right)=\pi_{r}\left(p_{r}^{H}, \theta^{L}, p_{d}^{H}\left(p_{r}^{H}\right) \mid \eta^{L}\right)-\pi_{r}\left(p_{r}^{L}, \theta^{L}, p_{d}^{L}\left(p_{r}^{L}\right), \mid \eta^{L}\right),
$$

and substituting (16)-(21) into $\Delta \pi_{r}\left(\eta^{L}\right)$, and obtained

$$
\Delta \pi_{r}\left(\eta^{L}\right)=-\frac{8 \alpha_{d} r^{4}\left(2 \alpha_{r} \alpha_{d}-\beta^{2}\right)\left(\eta^{H}-\eta^{L}\right)^{2} \Sigma_{L}^{2}}{\left[8 \alpha_{d} \eta^{H}-\gamma^{2}\left(2 \alpha_{r} \alpha_{d}-\beta^{2}\right)\right]^{2}\left[8 \alpha_{d} \eta^{L}-\gamma^{2}\left(2 \alpha_{r} \alpha_{d}-\beta^{2}\right)\right]^{2}} .
$$

where $\Sigma_{L}=2 A \alpha_{d}(1-\gamma)+\beta^{2}\left(2 w+c_{r}\right)+A \beta(\rho+\gamma)-2 \alpha_{d} \alpha_{r}\left(w+c_{r}\right)$.

Therefore

If $\Sigma_{L} \neq 0$, then $\Delta \pi_{r}\left(\eta^{L}\right)<0$, the retailer with low innovation cost will choose $p_{r}^{L}$;

If $\Sigma_{L}=0$, then $\Delta \pi_{r}\left(\eta^{L}\right)=0$, there is no difference in the two policies of the retailer with low innovation cost.

Sum up the above mentioned two situations; we know that there are the following three possible perfect Bayesian Nash equilibrium of the dynamic game model with asymmetric information.

(1) When $\Sigma_{H}<0$ and $\Sigma_{L} \neq 0$, the equilibrium solution of the game is separating equilibrium, the retailer with high innovation cost will choose $p_{r}^{H}$, the retailer with low innovation cost will choose $p_{r}^{L}$, and the manufacturer will choose the policy corresponding to the true information, in this case, the manufacturer and the retailer's profits of the dual channel supply chain is the same as that of perfect information, the manufacturer's posterior probability $\tilde{\mu}\left(\eta^{H} \mid p_{r}^{H}\right)=1, \tilde{\mu}\left(\eta^{L} \mid p_{r}^{H}\right)=0, \tilde{\mu}\left(\eta^{L} \mid p_{r}^{L}\right)=1$, 
$\tilde{\mu}\left(\eta^{H} \mid p_{r}^{L}\right)=0$

(2) When $\Sigma_{H}>0$ and $\Sigma_{L} \neq 0$, the equilibrium solution of the game is pooling

equilibrium, no matter the type of retailer's innovation cost, the retailer will always choose $p_{r}^{L}$, the manufacturer's posterior probability $\tilde{\mu}\left(\eta^{H} \mid p_{r}^{H}\right)=\mu\left(\eta^{H}\right), \tilde{\mu}\left(\eta^{L} \mid p_{r}^{H}\right)$ $=\mu\left(\eta^{L}\right), \tilde{\mu}\left(\eta^{L} \mid p_{r}^{L}\right)=\mu\left(\eta^{L}\right)$, and $\tilde{\mu}\left(\eta^{H} \mid p_{r}^{L}\right)=\mu\left(\eta^{H}\right)$.

(3) When $\Sigma_{H}=0$ and $\Sigma_{L} \neq 0$, the equilibrium solution of the game is semi separating equilibrium, the retailer with high innovation cost will random choose $p_{r}^{H}$ and $p_{r}^{L}$, the retailer with low innovation cost will choose $p_{r}^{L}$, the manufacturer's posterior probability $\tilde{\mu}\left(\eta^{H} \mid p_{r}^{H}\right)=1, \tilde{\mu}\left(\eta^{H} \mid p_{r}^{L}\right)<\mu\left(\eta^{H}\right), \tilde{\mu}\left(\eta^{L} \mid p_{r}^{L}\right)>\mu\left(\eta^{L}\right)$.

(4) When $\Sigma_{H}<0$ and $\Sigma_{L}=0$, the equilibrium solution of the game is semi separating equilibrium, the retailer with high innovation cost will choose $p_{r}^{H}$, the retailer with low innovation cost will random choose $p_{r}^{L}$ and $p_{r}^{H}$, the manufacturer's posterior probability $\tilde{\mu}\left(\eta^{L} \mid p_{r}^{L}\right)=1, \quad \tilde{\mu}\left(\eta^{H} \mid p_{r}^{H}\right)<\mu\left(\eta^{H}\right), \tilde{\mu}\left(\eta^{L} \mid p_{r}^{H}\right)>\mu\left(\eta^{L}\right)$.

(5) When $\Sigma_{H}>0$ and $\Sigma_{L}=0$, the equilibrium solution of the game is semi separating equilibrium, the retailer with high innovation cost will choose $p_{r}^{L}$, the retailer with low innovation cost will random choose $p_{r}^{L}$ and $p_{r}^{H}$, the manufacturer's posterior probability $\tilde{\mu}\left(\eta^{L} \mid p_{r}^{H}\right)=1, \tilde{\mu}\left(\eta^{H} \mid p_{r}^{L}\right)<\mu\left(\eta^{H}\right), \tilde{\mu}\left(\eta^{L} \mid p_{r}^{L}\right)>\mu\left(\eta^{L}\right)$.

(6) When $\Sigma_{H}=0$ and $\Sigma_{L}=0$, the equilibrium solution of the game is pooling

equilibrium, no matter the type of retailer's innovation cost, the retailer will random choose $p_{r}^{L}$ and $p_{r}^{H}$, the manufacturer's posterior probability $\tilde{\mu}\left(\eta^{H} \mid p_{r}^{H}\right)=\mu\left(\eta^{H}\right)$, $\tilde{\mu}\left(\eta^{L} \mid p_{r}^{H}\right)=\mu\left(\eta^{L}\right), \tilde{\mu}\left(\eta^{L} \mid p_{r}^{L}\right)=\mu\left(\eta^{L}\right)$, and $\tilde{\mu}\left(\eta^{H} \mid p_{r}^{L}\right)=\mu\left(\eta^{H}\right)$.

From the analysis of the manufacturer and retailer's pricing strategy under the condition of retailer's innovation cost type is asymmetric information, we know that the manufacturer's optimal pricing strategy is consistent with the type of retailer's optimal pricing strategy, when the retailer make decisions, there is no need to think about the condition of manufacturer possibly change the strategy, because for the rational manufacturer, choosing the pricing strategy consistent with the retailer is optimal to him, any behaviors of changing pricing strategy are all irrational to manufacturer, and it is unbelievable to the retailer. As long as the retailer calculates his profits according to different parameters, he can choose the corresponding action to ensure the maximum benefit.

\section{Concluding Remarks}

The article studied the dual channel supply chain system which are composed of one manufacture and retailer, and sells a single product, and the retailer is the leader, it mainly discussed the manufacturer and retailer's optimal pricing strategy under the condition of retailer's innovation cost coefficient is common knowledge and private information, and draw the conclusion that no matter how the manufacturer correct his belief on the 
retailer's private information, the manufacturer's optimal pricing strategy is always consistent with the retailer's optimal pricing strategy, while the retailer's optimal decision exists many cases, in asymmetric information case, the perfect Bayesian Nash equilibrium of the dynamic game model possibly exist various equilibrium solution, the difference of equilibrium solution depends on whether the retailer can obtain the more profits by concealing his private information, while the weak manufacturer only passively receive the retailer's decision. Consequently, under the condition of his innovation cost coefficient is his private information; the retailer's profit is always no less than that of perfect information, in other words, the retailer obtained the greater profit by asymmetric information.

There are several directions for further analysis that will achieve a better understanding of dual channel supply chain. For instance, one may consider the pricing or inventory strategy under the condition of the manufacturer and retailer's other information are all private information, in addition, it would be interesting to examine the situation of more manufactures and retailers, and it is insightful to consider the nonlinear demand function, the retailer's innovation cost coefficient is follows some specific distribution, dual channel supply chain's collaboration strategy and so on.

\section{Acknowledgments}

This work was supported by NSFC (11501525), Natural Science Foundation of Henan Province (152300410126), Project of Youth Backbone Teachers of Colleges in Henan Province (2015GGJS-179, 2013GGJS-142), Project of Youth Backbone Teachers of Colleges in Zhengzhou University of Aeronautics in 2015 and 2016, Fund of Aeronautics Science (2013ZD55006), Natural Science Foundation of Henan Education Department (16A630061, 14B110023).

\section{References}

[1] Wei-yu Kevin Chiang, "Product availability in competitive and cooperative dual-channel distribution with stock-out based substitution", European Journal of Operational Research., vol. 200, no. 2, (2010), pp.111-126.

[2] Webb K L, "Understanding hybrid channel conflict: a conceptual model and four case studies", Ann Arbor (MI): UMI Dissertation Services, (1997).

[3] Chiang W K, Chhajed P, Hess J D. "Direct marketing, indirect profits: a strategic analysis of dual channel supply chain design”, Manage Science., 2003, vol. 49, no. 2, (2003), pp.1-20.

[4] Cattanti K, Giland W, Hesse H S, "Boiling frogs: pricing strategies for a manufacturer adding a direct channel that competes with the traditional channel", Production and Operations Management., vol. 15, no. 1, (2006), pp.40-56.

[5] Aray A, Mittendorf B, Sappingtou D E M. "The bright side of supplier encroachment", Marketing Science., vol. 26, no. 5, (2007), pp.651-659.

[6] Cai G G, Zhang Z G, "Game theoretical perspectives on dual channel supply chain competition with price discounts and pricing schemes", International Journal of Production Economics., vol. 117, no. 1, (2009), pp.80-120.

[7] Zhang Xiaojuan, Wang Yong, "Dual channel supply chain decision with retailer's capital constraint", Control and Decision., vol. 29, no. 2, (2014), pp. 299-306.

[8] Shen Chengran, Xiong Zhongkai, Yan Wei, "Pricing and Coordination Research of Dual channel Supply Chain under Price Comparison”, Chinese Journal of Management Science., vol. 22, no. 1, (2014), pp. 84-93.

[9] Zeng Mingang, Wang Xuliang, "Pricing strategy of dual channel supply chain under demand uncertainty", Industrial engineering journal., vol. 16, no. 2, (2013), pp. 67-73.

[10] Fan Hehua, Zhou Yongwei, "Pricing and Inventory Strategy of Dual channel Supply Chain under Random Demand and Retailer's Capital Constraint", International Journal of Cognitive Informatics and Natural Intelligence., vol. 9, no. 1, (2015), pp. 30-46.

[11] Xu Min-li, Nie Xiao-zhe, Jian Hui-yun, "Pricing decision of dual channel supply chain with risk preference", Control and Decision., vol. 31, no. 2, (2016), pp. 238-245. 
[12] Zhou Yong-wei, Fan He-hua, Li Yan-chao, "Competition and coordination for the dual channel supply chain with channel preference", International Journal of u-and e-Service., Science and Technology, vol. 9, no. 2, (2016), pp. 49-58.

[13] Chen Jun, He Yuan, Lai Xin, "Service cooperation incentive mechanism in a dual channel supply chain under asymmetry information", Industrial Engineering Journal., vol. 17, no. 5, (2014), pp. 108-113.

[14] Tian Wei, Jiang Kan, "Wang Donghong, Supply chain effects of retailer's innovation investment for mixed channels under information asymmetry", Operations research and management science., vol. 23, no. 4, (2014), pp. 82-88.

[15] Wang Hong, Sun Yuling, Zhou Jing, "Pricing decision in dual channel supply chain under asymmetric manufacturer's risk averse information”, Operations research and management science., vol. 22, no. 6, (2013), pp. 117-122.

[16] Wang Hong, Ni Weitao, Zhou Jing, "Pricing decision in dual channel supply chain under asymmetric information", Chinese Journal of Management., vol. 7, no. 2, (2010), pp. 238-242.

[17] Chen shuzhen, Xiong Zhongkai, Li Gendao, "Coordination mechanism based on strategic innovative compensation in dual channel supply chains", Journal of Industrial engineering/engineering management., vol. 25, no. 2, (2011), pp. 45-53.

[18] Yan R, "Managing channel coordination in a multi-channel manufacturer-retailer supply chain”, Industrial Marketing Management., vol. 40, no. 2, (2011), pp. 636-642.

[19] Zhou Yong-wei, Fan He-hua, "Pricing Strategy of Dual channel Supply Chain under Random Demand and Joint Promotion", Statistics \& Decision., vol. 20, no. 18, (2015), pp. 51-55.

[20] Cho K R, Gerchak Y., "Supply chain coordination with downstream operating costs: coordination and investment to improve downstream operating efficiency", Europe Journal of Operational Research, vol. 162, no. 1, (2005), pp. 762-772.

[21] Zhang Lili, Wang Junmin, Geng Xiangping, "Application of Hesse Matrices in condition extreme value for multivariate function," Journal of Anyang Institute of technology., vol. 16, no. 18 , (2015), pp. $79-81$.

\section{Authors}

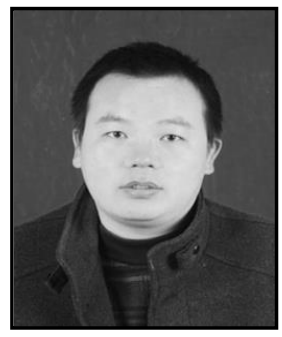

ZHOU Yong-wei, he received BS degree from Henan Normal University in 2000, the MS degree from Central South University in 2005, since 2011, he has been a full associate professor in Zhengzhou University of Aeronautics, his research interests include stochastic optimization and its application.

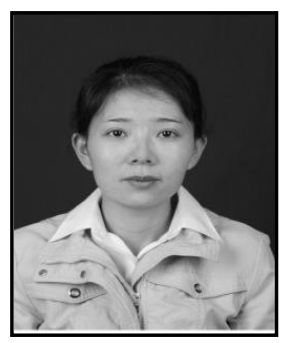

FAN He-hua, she received BS degree from Henan Normal University in 2000, the MS degree from Central South University in 2005, since 2016, she has been a full associate professor in Zhengzhou University of Aeronautics, her research interests include Markov process and its application and stochastic optimization theory. 